\title{
Omisión y desmemoria en la historia de los servicios bibliotecarios en la Argentina
}

Susana Romanos

Consejo Asesor de Información, cultura y sociedad: revista del Instituto de Investigaciones Bibliotecológicas. Universidad de Buenos Aires, Facultad de Filosofía y Letras | sromanos963@gmail.com / https://orcid.org/oooo-0002-7037-728X

\section{Resumen}

Luego de una breve introducción sobre la memoria, la omisión y el consiguiente olvido, se plantea la cuestión de la enseñanza de la historia de los servicios bibliotecarios en la Argentina a partir de mediados del siglo pasado, cuando se intentó implantar el modelo angloamericano como el único posible y el mejor, y se ignoraron los modelos preexistentes: tradicional e innovador. Se proponen interpretaciones basadas en

Palabras clave

Servicios al publico

Modelos

Historia

Argentina factores políticos, sociales y culturales.

\section{Omission and forgetfulness in the history of library services in Argentina}

\begin{abstract}
After a brief introduction on memory, omission and subsequent forgetfulness, the question of teaching the history of library services in Argentina from the middle of the last century arises, when it was attempted to implement the Anglo-American model as the only possible and the best, and pre-existing models were ignored: traditional and innovative. Interpretations based on political, social and cultural factors are proposed.
\end{abstract}

\section{Keywords}

Public services

Models

History

Argentina

Artículo recibido: 09-02-2020 Aceptado: 19-02-2020

\section{Introducción}

Una definición aceptada ampliamente de memoria humana es la que dice que es una función del cerebro que permite al ser humano adquirir, almacenar y recuperar información sobre distintos tipos de conocimientos, habilidades y experiencias pasadas. 
1. Basado en Romanos de Tiratel, Susana. Servicios al público: paradigmas, contradicciones y lectores. IX Jornadas Regionales y VII Jornadas Provinciales de Bibliotecarios "La biblioteca en el bicentenario y su proyección en el siglo XXI". Rosario, Biblioteca Argentina "Dr. Juan Álvarez" 10 y 11 de setiembre de 2010 y en Las trampas de la memoria. I Jornada de Referencia. Buenos Aires, Facultad de Ciencias Económicas, Universidad de Buenos Aires, 19 de septiembre de 2019).
Sin embargo, la memoria no es un sistema de almacenamiento perfecto. Aunque en muchas ocasiones se compare a la memoria humana con la capacidad de almacenamiento de una computadora, las diferencias se encuentran en su forma de recuperar los recuerdos o archivos guardados.

La computadora recupera un archivo sin ningún tipo de modificación o cambio, independientemente de cuando haya sido almacenado; mientras que los recuerdos recuperados de la memoria pueden verse alterados y modificados por muchos factores.

Los recuerdos pueden verse influidos por otros recuerdos, por la recepción de información nueva, por la interpretación que se pueda hacer de lo sucedido, por la propia creatividad, por la capacidad de invención...

También puede suceder que se modifiquen los recuerdos para que encajen con las expectativas o los intereses de los sujetos o de los colectivos humanos, obteniendo como resultado recuerdos que contienen errores y distorsiones o, simplemente, se nieguen hechos pretéritos, consciente o inconscientemente, haciendo lo que he dado en llamar las trampas de la memoria por omisión. Si lo que no se nombra no existe, los dispositivos de enseñanza pueden muy bien ir borrando la memoria del pasado logrando, de este modo, una amnesia generalizada. Cualquier disciplina de la que se ignora la historia puede verse como nueva. Un área que no documenta su pasado, carecerá de historia y también tendrá un sentido disminuido de identidad. Si ignoramos el pasado, seremos vistos continuamente como nuevos y emergentes (Buckland y Hahn. 1997: 285-286).

Dado que hace un poco más de 8 años que me retiré de la enseñanza y la investigación en la Facultad de Filosofía y Letras de la Universidad de Buenos Aires (FFL-UBA), ignoro qué historia de los servicios al público se enseña en la actualidad en la FFLUBA, solo recuerdo lo que me enseñaron a mí hace 40 años: una historia radicada exclusivamente en los Estados Unidos de Norteamérica, historia matizada hacia el final de mi carrera por una asignatura: la Documentación, surgida y desarrollada en la Europa continental, materia que, además, desapareció del plan actualmente vigente desde 2001, así como también del nombre del Departamento, con el agravante de que, si bien se reemplazó por Ciencia de la Información, no existe una sola materia dedicada a proporcionar los lineamientos básicos, los debates teóricos y el modelo dominante respecto de esa disciplina asociada a la Bibliotecología. En resumen, los egresados reciben un título, Licenciados en Bibliotecología y Ciencia de la Información, del que ignoran una parte sustancial o solo tienen una vaga idea al respecto.

El cuestionamiento, pues, surge espontáneamente ¿por qué? ¿qué sucedió para que lo enseñado respecto de los servicios al público en facultades e institutos sirviera, en gran parte, para desalentar otros modelos, otras tendencias, otras prácticas imperantes en la Argentina desde los inicios del siglo XX?

Lo que veremos a continuación será mi mirada - personal y discutible- de la amnesia que envolvió a la historia de los servicios en nuestro país y a sus posibles explicaciones.

\section{Los modelos de los servicios al público ${ }^{1}$}

En la Argentina, a mediados del siglo pasado se dio inicio a un cambio de modelo bibliotecológico cuya intención era reemplazar el vigente, que se había originado en Europa continental, por el angloamericano. Esta mutación comenzó en el campo de la enseñanza y, si bien nadie discutía las virtudes de uno de los modelos estadounidenses, anclado en la biblioteca pública, en la práctica de nuestro medio tuvo bajos niveles de implementación. La organización de las bibliotecas se regía, y en muchos 
casos aún sigue haciéndolo, en forma preponderante, por el modelo europeo de biblioteca para especialistas y eruditos, con estanterías cerradas, donde el catálogo es una forma de diálogo fundamental, casi único, para acceder a los materiales que posee la institución. Este esquema da por sentado un cierto nivel intelectual en los usuarios que les permita abstraer, a partir de las representaciones paratextuales de los materiales bibliotecarios, tal como figuran en los registros catalográficos, una realidad compartida, compleja, pero abordable para quienes tienen, al menos, una escolaridad de segundo ciclo. La disposición física de las plantas edilicias, del mostrador de préstamo y la entrega de libros mediada por el catálogo, entre otras cosas, acentúan la función de conservación y recogen una vieja tradición muy enraizada que le asigna un alto valor a las posesiones patrimoniales, en este caso, los materiales de las bibliotecas.

Tal como ya lo hemos mencionado, en el paquete angloamericano venía incluido un servicio al público que, desde ciertas corrientes de pensamiento, se presentó como el mejor cuando no el único posible. Me refiero al servicio de referencia, traducción fiel de los términos en inglés ${ }^{2}$. Charles A. Bunge lo define como la asistencia personal brindada a los usuarios en la obtención de información y agrega que el servicio de referencia tiene tres aspectos principales o técnicas de servicio: el servicio de información que consiste en encontrar la información que necesitan los usuarios o en asistir al usuario a encontrarla. La instrucción en el manejo de la biblioteca para ayudar a los usuarios a adquirir las habilidades necesarias para ubicar y usar los materiales informativos. Finalmente, la guía que asiste a los lectores en la elección de los ítems más adecuados para sus necesidades de educación, información o recreación (Bunge, 1980). La mayor parte de los historiadores estadounidenses del servicio de referencia encuentra conveniente comenzarlo con el ensayo de Samuel Swett Green de 1876, "Personal Relations Between Librarians and Readers", quien sugirió que el referencista debía desempeñar cuatro funciones: instruir al lector en el uso de la biblioteca; asistirlo en sus preguntas; ayudarlo en la selección de obras buenas; y promover la biblioteca dentro de la comunidad. Green señaló que los usuarios de la biblioteca no tenían habilidad en el uso del catálogo para encontrar los materiales y carecían del conocimiento necesario para seleccionar el material apropiado a sus necesidades. La asistencia personal a tales usuarios, dice Green, los beneficiaría y daría como resultado una visión más positiva de la biblioteca (Green, 1876). Las ideas de Green ganaron difusión, aceptación y apoyo. Para el año 1890, el término "trabajo o tarea de referencia" fue reemplazando a los primeros términos "ayuda a los lectores" y "asistencia a los lectores". Esto fue así, particularmente, en las bibliotecas públicas, donde, al declinar el siglo XIX ya estaban empleando personal de referencia idóneo. Los primeros años del siglo XX mostraron: 1) el establecimiento de departamentos de referencia separados en las bibliotecas públicas; 2) la aceptación de preguntas de referencia por teléfono o por correspondencia; 3) la extensión del servicio de referencia a las sucursales; y 4) la diferenciación de los servicios de referencia por niveles de complejidad y de materia. El desarrollo de los servicios de referencia fue más lento en las bibliotecas de los colleges superiores y de las universidades de postgrado que en las bibliotecas públicas y en las especializadas. Esto se debió, al menos en parte, a la presunción de que los miembros del profesorado y los estudiantes debían ser capaces de encontrar sus propios materiales y la información, y al énfasis que la comunidad académica colocó en el desarrollo de la colección y en el acceso por materia a través del catálogo (Bunge, 1980).

En la década del '40 del siglo pasado, este modelo de servicio se había impuesto en la organización de las bibliotecas de los EE. UU., combinado con estanterías abiertas y procesos técnicos centralizados. Es el momento en el cual Carlos V. Penna viaja a ese país y se entusiasma con la organización, la fuerza y la penetración de este sistema bibliotecario, implantado en una nación poderosa que, luego de terminada la
2. Esta expresión se impuso en la Argentina, en otros países de América Latina, como México, se prefirió servicio de consulta. En Italia y en Francia se habla de servicios de información. En este último país, el servicio de referencia es uno de los posibles servicios de información que presta la biblioteca. 
3. Fue cerrada en 1994 cuando, durante el gobierno del presidente Menem, se privatiza la Caja.

4. Es muy interesante analizar la bibliografía generada en las décadas iniciales del siglo pasado de lo que se dio en llamar la "Guerra de los decimalistas".
Segunda Guerra Mundial se perfilaba como una de las más ricas del mundo, indemne a los estragos de la contienda europea y con un discurso democrático interno muy persuasivo, aunque en su política externa no aplicara el mismo discurso y continuara una agresiva política de expansionismo económico en todo el mundo. Además, en el modelo a importar, se reflejaban de un modo transparente los valores básicos de la cultura estadounidense: la posición central del actor individual, la importancia de hacer que las cosas pasen y avancen a pesar de las barreras que se enfrentan y la confianza en las capacidades individuales para resolver problemas, valores con los que muy bien puede identificarse el liderazgo que ejerció Penna en sus colegas, sobre todo en Sabor a quien impulsó a enseñar Referencia, tal y como se hacía en Estados Unidos, cuando le entregó el clásico libro de Margaret Hutchins (1944) para que lo leyera, lo aprendiera y lo transmitiera en sus clases.

La situación en nuestro país de este grupo de bibliotecarios no era fácil, adherentes a un antiperonismo acrítico, varios de sus miembros fueron cesanteados de sus cargos en el Estado y se refugiaron en diversos trabajos, con una modalidad que, lamentablemente, se ha repetido en forma cíclica en nuestro país, la del exilio interno. Este conglomerado, no necesariamente parejo en cuanto a su formación, concentró una gran fuerza intelectual, asumió un liderazgo basado en la consistencia sin fisuras de un discurso democratizador, replegó su enseñanza en una universidad privada, la del Museo Social Argentino, que se convirtió en la usina donde se fue pergeñando el nuevo modelo bibliotecario. Es muy posible que, en una interpretación lineal y muy maniquea, los integrantes de ese cuerpo docente, a partir de sus propias convicciones políticas y de sus historias personales, hayan asimilado al movimiento justicialista con el fascismo europeo y a la democracia liberadora con los EE.UU. De ahí, solo hubo un paso, pequeño por cierto, alejarse de una tradición bibliotecaria nacional, de raigambre europea para apoyar a otro modelo que se presentaba como una herramienta para educar al pueblo y apartarlo, así, de falsos espejismos políticos. La estrategia para darlo a conocer destacando sus virtudes democratizadoras y civilizadoras fue implantarlo a través de la enseñanza por un lado y, por otro, ejemplificarlo mediante la aplicación a la organización de una biblioteca que fue la encarnación de la biblioteca pública según los principios angloamericanos, me refiero a la Biblioteca de la Caja de Ahorro 3 .

O sea, que cuando se trata de imponer el modelo de sistema bibliotecario angloamericano, en la Argentina ya existía un modelo europeo bien consolidado en las bibliotecas universitarias, en las especializadas y en las públicas, incluso con dos modelos en pugna: el tradicional del siglo XIX y el innovador, al que podríamos denominar movimiento bibliográfico-documental, iniciado a principios del siglo pasado e inspirado en los principios del Institut International de Bibliographie, fundado por Paul Otlet y Henri Lafontaine ${ }^{4}$. Los catálogos sistemáticos de gran parte de las bibliotecas de las universidades nacionales son prueba de ello, los centros de documentación e información anexos o independientes también lo son.

En forma recurrente se ha ignorado la riqueza del pensamiento bibliotecario argentino de la primera mitad del siglo pasado. Figuras activas hasta 1930, como Luis Ricardo Fors (Pineda, 1843-Buenos Aires, 1915), Juan Túmburus (Cormons (Trieste), 1861- Buenos Aires, 1929) -como exponentes de la corriente tradicional-y Federico Birabén (Buenos Aires 1867-Buenos Aires 1929) -representante del pensamiento innovador- aun cuando sostuvieron puntos de vista en conflicto respecto de la organización de las bibliotecas, compartieron conceptos en relación con los servicios: la centralidad del lector y de sus demandas como justificación social de la existencia de las bibliotecas. Por otra parte, aunque tímidamente, se va instalando el concepto del préstamo a domicilio, sobre todo en bibliotecas ligadas a centros de enseñanza. El más conservador y escueto en este ítem es Ricardo Fors (1905); un año antes, Birabén 
(1904) sostiene, sin decaer un solo momento, que el quid, el servicio esencial de una biblioteca es el bibliográfico. Para cumplimentarlo hay que elaborar repertorios y adquirir las obras que sean necesarias. Túmburus (1913) agrega, para bibliotecas de mayor envergadura, no solo el servicio de búsqueda en los catálogos y de provisión de los registros en fichas (se limita a lo que posee en texto completo), sino también el servicio de alerta a través de publicaciones ad hoc y el de traducciones.

La ordenación de los libros en los estantes se relaciona con la mayor o menor libertad de acceso y de movimiento de los lectores. Si la lectura en sala está concebida como un almacén, con un mostrador de despacho, a cuyo depósito solo tiene acceso el bibliotecario, los materiales pueden ordenarse por accesión, con signatura fija, o por tamaño a través de dos instancias de trabajo, con la signatura asignada primero en borrador hasta completar el anaquel y luego definitiva cuando ya se lo ha hecho. Todos los autores incluidos dentro de la corriente tradicional sustentan esta imagen espacial de la biblioteca y de la relación mediada, en forma directa, entre el usuario y los materiales. Acá destaca y se separa de un modo muy innovador para la época, el representante de la corriente renovadora porque, muy pronto, casi al inicio del siglo (Birabén, 1904), propone el agrupamiento de los materiales por temas en los estantes, propuesta inspirada en Paul Otlet porque, como dice Birabén para eso fue creada la clasificación decimal -sin percibir, quizás, la dimensión teórica que implica su comentario5. En este caso existe otra representación espacial de la biblioteca, más en consonancia con lo que son los supermercados actuales, donde un usuario activo recorre las estanterías y descubre no solo lo que requiere sino, además, material asociado que no había supuesto pudiera existir. Se sienta, lee, toma nota, busca de nuevo. Si no pide ayuda en forma explícita, nadie lo perturba. A diferencia de la anterior, la relación mediada es indirecta y casi transparente para el lector.

Otra prueba irrefutable de la pervivencia de la influencia europea se puede ver en la enseñanza de la Documentación y en la denominación de nuestras carreras hasta hace unos pocos años. Esta disciplina, de larga tradición e historia en la Argentina (ya en el plan de 1955, en la Facultad de Filosofía y Letras de la Universidad de Buenos Aires, existía una asignatura denominada Bibliografía y Documentación) se sostiene como una vía paralela por donde transita la problemática de la transferencia de la información científica. En la Carrera de Bibliotecarios de la FFL-UBA ${ }^{6}$ la disciplina fue directamente introducida desde Francia donde, Roberto Juarroz, becado por concurso por la Universidad de Buenos Aires para realizar estudios de perfeccionamiento, había residido en París entre 1961-1962. Allí asistió a cursos en la Sorbona, universidad en la que obtuvo el diploma superior de bibliotecario y tuvo la fortuna de conocer y ampliar sus conocimientos sobre Bibliografía con Louise-Noëlle Malclès. Además, estudió Documentación en el Institut National des Techniques de la Documentation. Con ese bagaje volvió a su país e inauguró, en la carrera de Filosofía y Letras de la UBA, en 1962, la cátedra de Documentación, haciéndose cargo, además, del dictado de la asignatura que se separa de la antes mencionada Bibliografía y Documentación. Juarroz es coherente en su postura y concibe siempre a la Documentación como disciplina autónoma e independiente de la Bibliotecología, tanto al publicar folletos separados (Juarroz, 1966 y Juarroz, 1984) como cuando, en 1966 le responde, en una muy interesante y jugosa confrontación teórica, a Josefa E. Sabor en relación con la autonomía disciplinaria de la Documentación (Sabor y Juarroz, 1966).

El marco teórico franco-belga, muy influyente en lo bibliográfico-documental, innovador en su momento, se pierde definitivamente y muy a destiempo, cuando el neoliberalismo en la Argentina declina y muestra una incapacidad notoria para solucionar los problemas políticos, sociales y económicos (2001). Es cuando se deja de enseñar Documentación y se cambia la denominación de las carreras a Bibliotecología y Ciencia de la Información.
5. En un trabajo, J. Frederic Finó y Luis A. Hourcade, deslizan un error algo incomprensible, dado que ambos citan y alaban este artículo de Birabén, publicado en 1904 pero, sin embargo, afirman que recién en 1932, por primera vez en la Argentina, Pedro B. Franco “... defiende la ubicación de los libros en el estante "por materias"" (Finó y Hourcade, 1952: 270, 273).

6. 1922-1957: Carrera de Bibliotecarios, dependiente de Biblioteca Central. 1958-1969. Carrera de Bibliotecarios dependiente del Departamento Bibliográfico. 1970-1974: Carrera de Ciencias de la Información dependiente del Departamento de Ciencias de la Información. 1975-1976: Carrera de Bibliotecología y Documentación dependiente de la Sección de Bibliotecología y Documentación del Departamento de Letras. 19762000: Carrera de Bibliotecología y Documentación dependiente del Departamento de Bibliotecología y Documentación. 2001- Carrera de Bibliotecología y Ciencia de la Información dependiente del Departamento de Bibliotecología y Ciencia de la Información. 
7. Baste recordar la obra de Louise-Noëlle Malclès, Les sources du travail bibliographique (Genève: Droz, 1950-1958, 4 v.) o el Bulletin de documentation bibliographique publicado por la Bibliothèque Nationale de France.
Hasta su despegue definitivo y espectacular del último cuarto del siglo anterior, los países de la Comunidad Europea presentaban una escala y unos procedimientos mucho más compatibles con las realidades latinoamericanas y se centraban más en las bibliotecas universitarias y especializadas que en las públicas. Repasemos a modo de ejemplo tres países europeos.

En Alemania, el servicio de información al público se desarrolló en las últimas décadas del siglo pasado en relación con el incremento del servicio de préstamo y con la liberalización para los usuarios del acceso al catálogo. El aumento de la frecuencia y la consecuente demanda creciente del material impusieron, primero por la vía informal, la exigencia de destinar parte del personal a la asistencia de los lectores. Se debe destacar que, al principio, se trató casi exclusivamente de la ayuda bibliográfica en el uso del catálogo sistemático, uno de los grandes filones del desarrollo de la Bibliotecología alemana. En síntesis, en las bibliotecas de este país la información a los lectores se estructuraba entonces de modo difuso en los distintos sectores de la biblioteca, como el escritorio de circulación, la sala de lectura y las secciones especiales (Aghemo, 1992: 14-15).

En Francia, el difundido manual de Richter en su edición de 1982, le presta escasa atención al problema del servicio de información que se debe prestar en las bibliotecas; en este país, en efecto, se cuidó más el aspecto bibliográfico, ya fuera para constituir el aparato de consulta o para producir instrumentos bibliográficos? ${ }^{7}$. Una señal de cambio y un testimonio imponente del nuevo interés por el aspecto de la biblioteca como centro informativo es, sin duda, la apertura al público en 1977 de la Bibliothèque Publique d'Information. Esos años y los de la siguiente década fueron muy revolucionarios para las bibliotecas francesas, lo testimonia la implantación del plan rector para la renovación y reorganización de las bibliotecas universitarias, la definición de una estructura de bibliotecas públicas regionales y la aparición de manuales de enseñanza que fijaron conceptos para la enseñanza y para la práctica profesional.

Las bibliotecas italianas, en el transcurso del siglo XX, tendían a orientarse hacia una dirección erudita mientras que las otras actividades, entre ellas la lectura, se dejaban a un bibliotecario subalterno considerado de rango inferior. Aghemo (1992: 16) da el ejemplo de una clasificación de las bibliotecas de 1939 que las dividía en "bibliotecas de alta cultura" y "bibliotecas de cultura común o general". Luego de la Segunda Guerra Mundial, la atención se centró en la problemática de la catalogación, de la adquisición, de la lectura y del préstamo. A fines de 1970 y principios de 1980 se desarrolló un gran interés en la cuestión bibliográfica (Aghemo, 1992: 17). Recién en la última década del siglo pasado se comenzó a identificar y promover la actividad de informar en la biblioteca como un servicio constituido como tal, dotado del personal especializado que sabe sacar lo mejor de todo el sistema informativo local donde se ubica la biblioteca y del externo con el que se relaciona (Aghemo, 1992: 19).

\section{Conclusiones}

Podemos concluir, entonces, que las características organizativas preponderantes en nuestro país dificultaron la implementación del modelo angloamericano. Entre las más destacadas podemos mencionar la diferencia de escala entre un país altamente desarrollado, que considera que una colección de dos millones de volúmenes para una biblioteca universitaria es pequeña, que tiene una organización administrativa departamental, con alta segregación de funciones, por ejemplo, la creación de departamentos por áreas disciplinarias y la abundancia de profesionales especializados en procedimientos técnicos, servicios de referencia, servicios automatizados, búsquedas 
en línea, etc., y una asignación presupuestaria suficiente para desarrollar los servicios con holgura, con bibliotecas públicas centrales, urbanas, poderosas, con gran cantidad de sucursales, con el problema de los procedimientos técnicos resuelto mediante la economía de esfuerzos representada primero por las fichas de la Library of Congress y luego por la catalogación compartida de las grandes redes bibliográficas conectadas electrónicamente, con edificios acordes con la idea misma de acceso abierto. Y una nación como la Argentina donde las condiciones de realización y de producción son otras, las colecciones son pequeñas -muy difícilmente algunas alcancen el millón de volúmenes, la organización administrativa no es departamental ni especializada porque no hay suficiente cantidad de bibliotecarios, por lo tanto, estos se ven exigidos por una multiplicidad de tareas diversas y, en la mayoría de los casos, no hay una partida anual asignada por la institución mayor para la biblioteca, sin un sistema de bibliotecas públicas potente, sin resolver aun la cuestión de los procedimientos técnicos que lleva a cada biblioteca argentina a realizar una y otra vez la catalogación y la clasificación del mismo ítem, con edificios pensados para albergar las colecciones detrás del escritorio de circulación, en ubicaciones fijas para ahorrar dinero y metros cuadrados cubiertos.

En la Argentina, las principales figuras representativas de la Bibliotecología, activos a mitad del siglo pasado, construyeron para sí mismos la imagen de fundadores sin pasado. A partir de esos años, desde los ámbitos superiores de enseñanza de la disciplina, se trató de imponer un modelo diferente, el angloamericano, y se lo presentó casi como la opción mejor para organizar nuestras bibliotecas, dándonos la idea, a quienes nos iniciábamos, de que antes el caos y la improvisación eran la piedra de toque reinante en dichos dispositivos culturales. La historia que se impartió, cuando todavía se lo hacía, fue la historia de las distintas ramas de especialización de la disciplina en los Estados Unidos. Sin embargo, tal como hemos podido apreciar en este trabajo, los debates dentro de la línea más clásica eran riquísimos, fundados en una larga tradición humanística creada en el Viejo Continente, base y sustento de nuestra propia cultura. Y la renovación, cuando llegó, tuvo un ímpetu creativo y militante admirable, aunque se haya circunscripto a las bibliotecas de investigación, fueran universitarias o especializadas.

La reflexión última, que asumo como propia, es si la relativa precariedad del sistema bibliotecario nacional no podría explicarse por la negación sistemática de una tradición de raíz europea que acompaña a la Argentina desde el siglo XVIII. Si acaso, en este recóndito campo del conocimiento, una vez más, las trampas de la memoria y la amnesia sistemática de las posiciones políticas involucradas en muchas de las decisiones de nuestra disciplina, ya sean en el mero ejercicio como en la enseñanza y la investigación, no se constituyen en un componente de parálisis y de confusión, y en una distorsión que desdibuja y frena la construcción colectiva de nuestra propia identidad.

\section{Agradecimientos}

Agradezco al Secretario de redacción, Dr. Alejandro E. Parada, y al Consejo Editorial por invitarme a publicar este trabajo en Información, cultura y sociedad. 


\section{Q Referencias bibliográficas}

》Aghemo, Aurelio. 1992. Informare in biblioteca. Milano: Editrice Bibliografica. 163 p. (Bibliografia e Biblioteconomia; 43)

"Birabén, Federico. 1904. La futura biblioteca universitaria: lo que podría ser. Revista de la Universidad de Buenos Aires. Vol. 1, no. 3, 232-247, no. 4,344-370 y no. 5, 451-489.

» Buckland, Michael K. y Trudi Bellardo Hahn. 1997. Introduction. En Journal of American Society for Information Science. Vol. 48, no. 4, 285-288.

»Bunge, Charles A. 1980. Reference services. En: ALA World encyclopedia of library information services. Chicago: American Library Association.

»Finó, J. Frederic y Luis A. Hourcade. 1952. Evolución de la Bibliotecología en la Argentina: 1757-1952. Universidad. Vol. 25, 265-301.

»Fors, Luis Ricardo. 1905. Biblionomía. La Plata. MS. 391 h. enc. en 2 v., 192 h., 193391 h.En el primer volumen falta el folio 193 que se encuadernó en el segundo volumen entre los folios 226 y 227.

»Green, Samuel Swett. 1876. "Personal Relations Between Librarians and Readers" En Library Journal, octubre, 74-81.

» Hutchins, Margaret. 1944. Introduction to Reference Work. Chicago: American Library Association.

» Juarroz, Roberto. 1966. Documentación. Buenos Aires: Centro Nacional de Documentación e Información Educativa.

» Juarroz, Roberto. 1984. Las Ciencias de la Información. Buenos Aires: Centro de Investigaciones Bibliotecológicas de la Facultad de Filosofía y Letras. 10 p. (Cuadernos de Bibliotecología; no. 7)

» Richter, B. 1982. Précis de bibliothéconomie. 3. éd. corr. et aum. München: K. G. Saur, $227 \mathrm{p}$.

"Romanos de Tiratel, Susana. 2010. Servicios al público. En Barber, Elsa E., comp. Aportes preliminares para el estudio de la Bibliotecología y Ciencia de la Información. Buenos Aires: Editorial de la Facultad de Filosofía y Letras, Universidad de Buenos Aires. p. 107-127 (Libros de Filo).

»Sabor, Josefa E. y Roberto Juarroz. 1966. Bibliotecología y Documentación. Buenos Aires: Centro Nacional de Documentación e Información Educativa.

» Túmburus, Juan. 1913. Apuntes de Bibliotecografía: notas histórico-bibliográficas sobre clasificación. Buenos Aires: Imprenta de Coni Hermanos. 16o p. 